\title{
A Key Recovery Attack on Discrete Log-based Schemes Using a Prime Order Subgroup ${ }^{\star}$
}

\author{
Chae Hoon Lim ${ }^{1}$ and Pil Joong Lee ${ }^{2}$ \\ 1 Information and Communications Research Ceater, Future Systems, Inc., 372-2, \\ Yang Jae-Dong, Seo Cho-Gu, Seoul, 137-130, KOREA \\ E-mail: chlim@future.co.kr \\ 2 Dept. of Electronic and Electrical Engineering, Pohang University of Science and \\ Technology (POSTECH), Pohang, 790-784, KOREA \\ E-mail: pjl@postech.ac.kr
}

\begin{abstract}
Consider the well-known oracle attack: somehow one gets a certain computation result as a function of a secret key from the secret key owner and tries to extract some information on the secret key. This attacking scenario is well understood in the cryptographic community. However, there are many protocols based on the discrete logarithm problem that turn out to leak many of the secret key bits from this oracle attack, unless suitable checkings are carried out. In this paper we present a key recovery attack on various discrete log-based schemes working in a prime order subgroup. Our attack may reveal part of, or the whole secret key in most Diffie-Hellman-type key exchange protocols and some applications of ElGamal encryption and signature schemes.
\end{abstract}

\section{Introduction}

Many cryptographic protocols have been developed based on the discrete logarithm problem. The main objective of developers is to design a protocol that is as difficult to break as the underlying discrete logarithm problem under some reasonable assumptions. On the other hand, the goal of attackers is to find a way to extract the secret key involved or to pretend to be a legitimate user without knowing the secret key. Though provable security guarantees that there is no efficient attack on the protocol, it should be carefully interpreted for practical security; the most important would be to use secure parameters and follow the assumed conditions or requirements as closely as possible. As an illustrative example, we refer to two recent papers on ElGamal-type signature schemes; one regarding security proof by Pointcheval and Stern [34] and the other regarding signature forgery by Bleichenbacher [4] (see also Stern [39] for further discussions on their apparent contradiction).

The purpose of this paper is to point out the insecurity of various discrete log-based schemes using a prime order subgroup. More specifically, we present a

\footnotetext{
* This work was done while the first author was in POSTECH Information Research Laboratories, POSTECH, Pohang, Korea and the second author was in NEC Research Institute, Princeton, NJ, during his sabbatical leave.
} 
key recovery attack on these protocols, which can find all or part of the secret key bits. Our attack is closely related to the choice of parameters and the checking of protocol variables. Thus, as is usual, our attack, once identified, can be easily prevented by adding suitable checking steps or by using 'secure' parameters. Here 'secure' means that the parameters are secure against our attack. And this also implies that the usual parameters commonly used in the literature are not secure against our attack. The presented attack demonstrates the importance of checking protocol variables in designing discrete log-based schemes.

Pohlig-Hellman Decomposition and Pollard's Methods : The discrete logarithm problem over $\mathbf{Z}_{p}^{*}$ can be broken down into a number of small such sub-problems defined over small order subgroups of $\mathbf{Z}_{p}^{*}$ (Pohlig-Hellman decomposition [33]). Then these sub-problems can be solved using Pollard's rho and lambda methods [35] and the resulting partial logarithms can be combined using the Chinese Remainder Theorem (CRT) to give the pursued discrete logarithm.

For simplicity, suppose that $p-1=\prod_{i=1}^{n} q_{i}\left(q_{i}\right.$ prime). Let $\alpha$ be a generator of $\mathbf{Z}_{p}^{*}$. Given $y=\alpha^{x} \bmod p$, we can reduce the problem of finding $x \bmod p-1$ to the following sub-problems: find $x_{i}=x \bmod q_{i}$ from $y_{i}=y^{(p-1) / q_{i}}=\alpha_{i}^{x_{i}} \bmod p$ for each $i$, where $\alpha_{i}=\alpha^{(p-1) / q_{i}}$ (an element of order $q_{i}$ ). Each such sub-problem can be solved using Pollard's rho method (see [40] for linear speedup with multiple processors). Once $x_{i}$ 's are found for all $i$, they can be combined using the CRT to yield the logarithm $x$ mod $p-1$. Pollard's rho method can compute a logarithm in a subgroup of prime order $q$ in time $O(\sqrt{q})$, while Pollard's lambda method can compute a logarithm that is known to lie within some restricted interval of width $w$ in time $O(\sqrt{w})$. Thus, both methods have similar square-root running time for a given size of an unknown exponent. In particular, the lambda method is very useful for computing a logarithm in a prime order subgroup when part of the logarithm is known. (For details, see van Oorschot and Wiener [41].)

The Attacking Scenario : In this paper we pay our attention to DL-based schemes using a prime order subgroup. Thus, as is usual, we assume that a prime $p$ is chosen at random such that $p-1$ has a large prime factor $q$. Let $g$ be an element of order $q$ and $\operatorname{ord}(\beta)$ denote the order of $\beta \bmod p$. Then, for a given $y$ such that $y=g^{x} \bmod p$, it is completely infeasible under current technology to find $x$ using Pollard's rho method, if we take for example $|q|=160$, since it requires about $2^{80}$ operations. Our observation is that if we could obtain $z=\gamma^{x} \bmod$ $p$ somehow by attacking a protocol, where $\gamma=\prod \beta_{i}$ (a product of distinct smooth order elements mod $p$ ), then we could find $x$ modulo $\operatorname{ord}(\gamma)$ using the Pohlig-Hellman decomposition. Here we assume that $(p-1) / q$ may have many small prime factors, which is usually the case for a randomly chosen prime $p$. And finally the remaining part of $x$ could be found from the public key $y$ using Pollard's lambda method. A special case of the attack is to find $x \bmod \operatorname{ord}(\beta)$, given $z=f\left(\beta^{x} \bmod p\right)$ for any function $f$. In this case, one can find $j=x \bmod$ $\operatorname{ord}(\beta)$ by checking that $z=f\left(\beta^{j} \bmod p\right)$ for $j=0,1, \cdots, \operatorname{ord}(\beta)-1$, where we assume that the range of $f$ is large enough compared to $\beta$, so that the probability 
of collisions occurring under $f$ is negligible, which is usually the case in most instances of our attack.

The main problem in the above attacking scenario is how to obtain a PohligHellman decomposition for the secret key. This should be impossible in welldesigned protocols. However, we could find many DL-based schemes susceptible to the above attack in the literature. Most Diffie-Hellman type key exchange protocols are vulnerable to the above attack. Other examples include shared decryption of ElGamal encryption, shared verification of ElGamal signatures and undeniable signatures. Our attack was possible in all these schemes, since the involved parties do not check relevant protocol variables. Though there are several papers pointing out the importance of checking public parameters and protocol variables (e.g., see $[4,42,1,41,2])$ in $\mathrm{DH}$ key exchange and digital signature schemes, no literature addresses such an explicit attack revealing the involved secret. Our attack may find the whole secret key in many cases.

Related Work : Previous work most relevant to our attack is the middleperson attack on the original Diffie-Hellman key exchange protocol [16] (see $[41,2]$ ). Two parties $A$ and $B$ agree on a prime $p$ and a generator $\alpha$ of $\mathbf{Z}_{p}^{*}$, exchange random exponentials, $r_{A}=\alpha^{k_{A}} \bmod p$ and $r_{B}=\alpha^{k_{B}} \bmod p$, and then compute a shared secret $K=r_{B}^{k_{A}}=r_{A}^{k_{B}}=\alpha^{k_{A} k_{B}} \bmod p$. Suppose that $p-1=q w$ with $w$ smooth. An attacker may replace $r_{A}$ and $r_{B}$ with $r_{A}^{q} \bmod p$ and $r_{B}^{q} \bmod p$ respectively. Then the shared key becomes $K=\left(\alpha^{q}\right)^{k_{A} k_{B}} \bmod p$, which can also be computed by the attacker since he can find $k_{i} \bmod w$ from $r_{i}$. This attack can be easily prevented by authenticating the random exchange, as in the STS [17] and SKEME [21] protocols. ${ }^{1}$

The above attack motivates the use of a prime order subgroup, which also substantially increases the efficiency in computation and parameter generation (see $[41,2]$ for further discussions). Thus most DL-based schemes have been designed using a prime order subgroup since its first invention by Schnorr [38]. However, this paper will show potential weaknesses in such a setting. Our attack on key exchange protocols is quite similar to the above attack, except that our target protocols use a prime order subgroup and that our objective is to find the long-term secret key of the involved party (usually by the other legitimate party). Our attack can be applied to any protocol involving a DH shared secret.

The rest of this paper is organized as follows: We present in Sec. 2 a key recovery attack on DH-type key exchange protocols and in Sec. 3 a similar attack on other DL-based schemes such as ElGamal encryption and signatures. Sec.4 deals with the generation of secure primes and public key certificates as possible countermeasures to minimize security loss by our attack. And we conclude in Sec.5.

${ }^{1}$ It is very important to authenticate the exchanged random messages themselves, rather than the shared secret computed from them. For example, the modified STS protocol by Boyd and Mao [5] may be vulnerable to the middleperson attack, since it only authenticates the hashed version of the shared secret. 


\section{Extracting Secret Keys in Key Exchange Protocols}

One of the well-known design principles for public key protocols states that a message received should not be assumed to have a particular form unless it can be checked [1]. In particular, it is very dangerous to apply one's secret to a number received from the other. However, this principle is hard to apply to Diffie-Hellman-type key exchange protocols. This has given rise to a lot of attacks or weaknesses under a variety of attacking scenarios. Most attacks aim at finding a session key (e.g., see $[10,43])$ or causing authentication failure (e.g., see [27]). In this section we present a key recovery attack that can be applied to many DH-type key exchange protocols published in the literature ${ }^{2}$ unless proper precautions are taken additionally.

\subsection{Basic Diffie-Hellman Key Exchange}

We first consider the case where a user $A$ successfully obtained a certificate on the public key $y_{A}=\beta g^{x_{A}} \bmod p$ with $\beta$ of small order $\bmod p$. This is possible unless a certification authority checks that $y_{A}^{q}=1 \bmod p$ before issuing a certificate for $y_{A}$. The CA usually requires that each user prove knowledge of a secret key corresponding to the public key to be certified, since otherwise there exist some protocols that can be attacked with a faked public key (e.g., see [27]). However, even in this case it may still be possible to register a public key of the form $y_{A}=\beta g^{x_{A}} \bmod p$ if it is not checked that $y_{A}^{q}=1 \bmod p$.

For example, suppose that for registration the CA requires a user's digital signature on the certificate message which contains all necessary information for certification, including the public key, as defined by X.509. In this case it is easy for $A$ to generate a valid signature corresponding to the public key $y_{A}=\beta g^{x_{A}}$ $\bmod p$ when $\operatorname{ord}(\beta)$ is small. For example, suppose that Schnorr's signature scheme [38] is used for this purpose. Given message $m, A$ can find $r^{\prime} \in(0, \operatorname{ord}(\beta)]$ such that $r^{\prime}=h\left({\beta^{\prime}}^{\prime} g^{k} \bmod p, m\right) \bmod \operatorname{ord}(\beta)$ in about $\operatorname{ord}(\beta)$ steps, where $k \in_{R} \mathbf{Z}_{q}$ and $h$ denotes a secure hash function. Thus $A$ can generate a signature $\{r, s\}$ on $m$ by computing $r=h\left(\beta^{r^{l}} g^{k} \bmod p, m\right)$ and $s=k-x r \bmod q$. It is easy to see that the resulting $\{r, s\}$ is a valid signature on $m$ with the public key $y_{A}=\beta g^{x_{A}}$ mod $p$. On the other hand, suppose that Schnorr's identification scheme is used instead. Then $A$ can pass the protocol with probability $1 / \operatorname{ord}(\beta)$ on average, irrespective of the size of a challenge by $B$ (A similar observation has been made before by Burmester [9]). Therefore, it is essential that the CA should first check that $y_{A}^{q}=1 \bmod p$.

${ }^{2}$ Diffie-Hellman-type key exchange protocols can be divided into two broad classes. The first is to exchange random exponentials and then authenticate the exchange using a separate authentication mechanism. The STS and SKEME protocols belong to this class. Such protocols seem to be the most robust against various attacks, including our one. Most other protocols involve the fixed secret/public key pair for key exchange and (possibly) authentication. Our attack can be applied to most of such protocols. 
We now present a key recovery attack under the assumption that an attacking user $i$ has a public key $y_{i}=\beta g^{x_{i}} \bmod p$. This attack will demonstrate the importance of the checking step in the certification process. We first consider the zero-message DH key exchange with fixed keys (e.g., used in [22] ${ }^{3}:$ Two users $A$ and $B$ share a session key $K$ by computing $K=h\left(y_{B}^{x_{A}} \bmod p, d\right)=$ $h\left(y_{A}^{x_{B}} \bmod p, d\right)$, where $d$ is time/date information. In this protocol, suppose that user $B$ with public key $y_{B}=g^{x_{B}} \bmod p$ uses a session key computed by $K=h\left(y_{A}^{x_{B}} \bmod p, d\right)$ to send a message $m$ to user $A$ with public key $y_{A}=\beta g^{x_{A}}$ $\bmod p$. Then, when receiving $\left\{c=E_{K}(m), d\right\}$ from $B, A$ can extract $|\operatorname{ord}(\beta)|$ bits of $B$ 's secret key by an exhaustive search. For this, $A$ computes $K_{j}=h\left(y_{B}^{\boldsymbol{x}_{A}} \cdot \beta^{j}\right.$ $\bmod p, d)$ for $0 \leq j<\operatorname{ord}(\beta)$, decrypts $c$ with each $K_{j}$ and checks that the result is a meaningful message. If a meaningful message is found for some $j$, then $A$ has found $j=x_{B} \bmod \operatorname{ord}(\beta)$.

User $A$ may repeat this attack by updating his public key with $\beta$ of a different order and combine the resulting partial secrets using the Chinese Remainder Theorem. This will give about $t$ bits of $x_{B}$ if $t$ is the bit-length of small prime factors of $p-1$ that can be used for this attack. Now the remaining $(|q|-t)$ bits of $x_{B}$ can be found in about $2^{(|q|-t) / 2}$ steps using Shanks' method or Pollard's lambda method (see [41] for further discussions). Note that if $\operatorname{ord}(\beta)$ is small, say of 20 bits, $A$ has little difficulty in reading the ciphertext directed to him. Also note that this attack can be applied to any protocol if the protocol reveals an equation involving the fixed DH key $g^{x_{A} x_{B}} \bmod p$.

As another example, let us consider the following non-interactive, symmetric protocol (modified from Protocol 2 in [27]).

1. $A$ computes $r_{A}=g^{k_{A}} \bmod p$ with $k_{A} \in \in_{R} \mathbf{Z}_{q}, K_{A 1}=y_{B}^{k_{A}} \bmod p, e_{A}=$ $h\left(K_{A 1}, r_{A}, A, B\right)$ and $s_{A}=k_{A}-x_{A} e_{A} \bmod q . A$ then sends $\left\{r_{A}, s_{A}\right\}$ to $B$.

2. $B$ computes $r_{B}=g^{k_{B}} \bmod p$ with $k_{B} \in \in_{R} \mathbf{Z}_{q}, K_{B 1}=y_{A}^{k_{B}} \bmod p, e_{B}=$ $h\left(K_{B 1}, r_{B}, B, A\right)$ and $s_{B}=k_{B}-x_{B} e_{B} \bmod q . B$ then sends $\left\{r_{B}, s_{B}\right\}$ to $A$.

3. $A$ computes $K_{A 2}=r_{B}^{x_{A}} \bmod p$ and $e_{B}=h\left(K_{A 2}, r_{B}, B, A\right)$, and checks that $g^{s_{B}} y_{B}^{e_{B}}=r_{B} \bmod p$. If the check succeeds, $A$ computes the session key $K_{A}=h\left(K_{A 1} K_{A 2} \bmod p\right)$. Otherwise, $A$ stops the protocol with failure.

4. $B$ computes $K_{B 2}=r_{A}^{x_{B}} \bmod p$ and $e_{A}=h\left(K_{B 2}, r_{A}, A, B\right)$, and checks that $g^{s_{A}} y_{A}^{e_{A}}=r_{A} \bmod p$. If the check succeeds, $B$ computes the session key $K_{B}=h\left(K_{B 1} K_{B 2} \bmod p\right)$. Otherwise, $B$ stops the protocol with failure.

Suppose that $A$ has a valid public key $y_{A}=\beta g^{x_{A}} \bmod p$. Then $A$ can find $j=k_{B} \bmod \operatorname{ord}(\beta)$ by checking that $g^{s_{B}} y_{B}^{e_{B}}=r_{B} \bmod p$ with $e_{B}=h\left(r_{B}^{x_{A}} \beta^{j}\right.$ $\left.\bmod p, r_{B}, B, A\right)$ for all possible values of $j$. This gives $|\operatorname{ord}(\beta)|$ bits of information on the secret key $x_{B}$, since $x_{B}=\left(s_{B}+k_{B}\right) e_{B}^{-1} \bmod q$, and thus the effective secret bits of $x_{B}$ is reduced to $(|q|-|o r d(\beta)|)$ bits. The reason why our attack can

${ }^{3}$ The SKIP protocol [3] being widely implemented in the industry for IP layer security also employs this scheme to get a long-term shared secret, which is used as a keyencrypting key. However, the SKIP documentation recommands to use a safe prime $p$, i.e., a prime $p$ such that $(p-1) / 2$ is also prime. Thus our attack on this protocol only discloses one bit of the secret, the parity bit. 
apply to this protocol is that the same random secret $k_{i}$ is used for authentication and session key computation. This shows that a robust protocol should avoid using the same secret (even if it is a one-time random number) for two different purposes [1]. In this respect the approach taken in the STS [17] and SKEME [21] seems to be a better way to design key exchange protocols.

\subsection{Authenticated Key Exchange}

The attack presented above can be easily prevented by a proper precaution in the certificate issuing process. We now extend our attack to the case where each user has a correct public key. As an example, we consider the following key exchange protocol, which is an authenticated version of the MTI (MatsumotoTakashima-Imai) protocol [26]. This protocol, with slight changes, is widely studied in the literature (e.g., see $[27,20])$ and is also being standardized in ISO/IEC JTC1/SC27 [44].

1. $A$ randomly picks $k_{A} \in \mathbf{Z}_{q}$, computes $r_{A}=g^{k_{A}} \bmod p$ and sends $r_{A}$ to $B$.

2. $B$ randomly picks $k_{B} \in \mathbf{Z}_{q}$, computes $r_{B}=g^{k_{B}} \bmod p, K_{B}=y_{A}^{k_{B}} r_{A}^{x_{B}} \bmod$ $p$ and $e_{B}=h\left(K_{B}, r_{B}, r_{A}, B, A\right)$, and sends $\left\{r_{B}, e_{B}\right\}$ to $A$.

3. $A$ computes $K_{A}=y_{B}^{k_{A}} r_{B}^{x_{A}} \bmod p$ and $e_{B}^{\prime}=h\left(K_{A}, r_{B}, r_{A}, B, A\right)$, and checks that $e_{B}=e_{B}^{\prime}$. If $e_{B} \neq e_{B}^{\prime}$, then $A$ stops the protocol with failure. (Optional) Otherwise, $A$ computes $e_{A}=h\left(K_{A}, r_{A}, r_{B}, A, B\right)$ and sends $e_{A}$ to $B$.

4. (Optional) $B$ computes $e_{A}^{\prime}=h\left(K_{B}, r_{A}, r_{B}, A, B\right)$ and checks that $e_{A}=e_{A}^{\prime}$. If $e_{A} \neq e_{A}^{\prime}$, then $B$ stops the protocol with failure.

The session key $K$ can be derived from the shared secret, for example, as $K=h\left(K_{A}\right)=h\left(K_{B}\right)$. The critical point relevant to our attack is the key authentication based on the shared secret $K_{A}=K_{B}$. That is, $B$ applies his secret key to the number received from $A$ and returns $e_{B}$ as a function of the (assumed) session key $K_{B}$. Suppose that $A$ sends $r_{A}=\beta g^{k_{A}} \bmod p$ in step 1. Then an honest user $B$ will compute $K_{B}=y_{A}^{k_{B}} r_{A}^{x_{B}}=r_{B}^{x_{A}} y_{B}^{k_{A}} \beta^{x_{B}} \bmod p$ and return $e_{B}$ computed with this $K_{B}$. Once receiving $\left\{r_{B}, e_{B}\right\}, A$ may abort the protocol if a response is required. Since $A$ can compute the first exponential in $K_{B}, y_{A}^{k_{B}}=r_{B}^{x_{A}} \bmod p$, it can find $j=x_{B} \bmod \operatorname{ord}(\beta)$ in $O\left(2^{\mid \operatorname{ord}(\beta) !}\right)$ steps by checking the equality $e_{B}=h\left(K_{B}, r_{B}, r_{A}, B, A\right)$ with $K_{B}=r_{B}^{x_{A}} y_{B}^{k_{A}} \beta^{j}$ for all possible values of $j$ (i.e., $j=0,1, \cdots, \operatorname{ord}(\beta)-1$ ).

The above attack may be repeated using different smooth order elements for which it is feasible to do the exhaustive search. Thus if $p-1$ has several prime factors of small size (say, less than 40 bits), then it would be possible to find the whole secret in reasonable time. We note that the attack can be mounted against any authenticated key exchange protocol as long as authentication is performed using the shared secret (note that such authentication is possible only if each user's secret key is involved in the computation of the shared secret). This implies that almost all key exchange protocols providing explicit authentication without using a separate authentication channel (e.g., as in STS [17] or SKEME [21]) may be vulnerable to our attack. 
Our attack can also be applied to key exchange protocols with implicit authentication, since the agreed upon session key will be used anyway in later communications. For example, suppose that user $A$ mounted the attack in the original MTI protocol, where each user exchanges random exponential $r_{i}$ and computes the session key as above. Now, if user $B$ first uses the resulting session key for message authentication (or key authentication), $A$ obtains a known equation involving the session key computed by $B$. Then the situation, in view of our attack, is the same as in the above authenticated protocol. On the other hand, if $B$ sends a ciphertext for an unknown message, then $A$ can find the intended partial secret by decrypting the ciphertext with all possible values of the session key that $B$ is supposed to compute and then finding a meaningful message. Note that usual known-key attacks assume knowledge of the whole shared secret from which the session key is derived (e.g., see $[43,10]$ ), but in our attack it is sufficient to obtain any function of the shared secret (even a ciphertext suffices).

We next show that some key exchange protocols using a signature scheme for authentication may also be vulnerable to our attack. For example, consider the following protocol (developed from Protocol 4 in [23]):

1. $A$ picks a random integer $k_{A} \in \mathbf{Z}_{q}$, computes $r_{A}=g^{k_{A}} \bmod p$ and sends $r_{A}$ to $B$.

2. $B$ picks a random integer $k_{B} \in \mathbf{Z}_{q}$, computes $r_{B}=g^{k_{B}} \bmod p$. $B$ also computes $K_{B}=r_{A}^{k_{B}} \bmod p, e_{B}=h\left(K_{B}, r_{B}, r_{A}, B, A\right)$ and $s_{B}=k_{B}-x_{B} e_{B}$ $\bmod q$, and sends $\left\{r_{B}, s_{B}\right\}$ to $A$.

3. $A$ computes $K_{A}=r_{B}^{k_{A}} \bmod p, e_{B}=h\left(K_{A}, r_{B}, r_{A}, B, A\right)$ and checks that $g^{s_{B}} y_{B}^{e_{B}}=r_{B} \bmod p$. If the check fails, then $A$ stops the protocol with failure. (Optional) Otherwise, $A$ computes $e_{A}=h\left(K_{A}, r_{A}, r_{B}, A, B\right)$ and $s_{A}=k_{A}-x_{A} e_{A} \bmod q$, and sends $s_{A}$ to $B$.

4. (Optional) $B$ computes $e_{A}=h\left(K_{B}, r_{A}, r_{B}, A, B\right)$ and checks that $g^{s_{A}} y_{A}^{e_{A}}=$ $r_{A} \bmod p$. If it does not hold, then $B$ stops the protocol with failure.

This protocol uses a digital signature on the shared secret $K_{A}=K_{B}=$ $g^{k_{A} k_{B}} \bmod p$ (for a honest run) to authenticate each other. However, the same random number is used for authentication and session key computation (as in the last example in Sec.2.1). This fact can be exploited by $A$ to extract partial information on the secret key $x_{B}$. As before, $A$ sends $r_{A}=\beta g^{k_{A}} \bmod p$ and does the exhaustive search for $k_{B} \bmod \operatorname{ord}(\beta)$ using the verification equation $g^{s_{B}} y_{B}^{h\left(K_{A}, r_{B}, r_{A}, B, A\right)}=r_{B} \bmod p$ with $K_{A}=r_{B}^{k_{A}} \beta^{k_{B}} \bmod p$. This reduces the effective secret bits of $x_{B}$ to $(|q|-|o r d(\beta)|)$ bits. Note, however, that repetition of the attack with $\beta$ of a different order does not help to find further bits of the secret in this case, since a different $k_{B}$ is used each time and $\operatorname{ord}(\beta)$ does not divide $q$.

The attack described in this section can be easily prevented by checking that $r_{i}^{q}=1 \bmod p$ for each random exponential exchanged before raising it to the secret key. However, this considerably increases the computational load. A better solution would be to choose a prime $p$ such that $(p-1) / 2 q$ has prime factors at least larger than $q$ (see Sec.4). Such a $p$ only leaks the parity bit of the secret 
key by our attack. Note that no key exchange protocol can protect the parity bit of the involved secret if the order of the received number is not checked as explained above, since there always exist an element of order 2 (i.e., p-1). This is also true for the following one-way key exchange protocol useful for email applications: $A$ computes $r_{A}=g^{k_{A}} \bmod p$ with random $k_{A} \in \mathbf{Z}_{q}$ and the session key $K=h\left(y_{B}^{k_{A}} \bmod p, r_{A}, d\right)$, encrypts a message $m$ as $c=E_{K}(m)$ and sends $\left\{r_{A}, d, c\right\}$ to $B$, where $d$ is a timestamp. $B$ can then compute $K=h\left(r_{A}^{x_{B}} \bmod \right.$ $\left.p, r_{A}, d\right)$ and decrypt $c$. In this protocol $A$ may send $r_{A}=-g^{k_{A}} \bmod p$. If $B$ does not respond or claims a garbage mail, then $A$ knows that $x_{B}$ is odd. This attack may be repeated $t$ times, revealing the last $t$ bits of $x_{B}$, if $2^{t} \mid p-1$.

\section{Extracting Secret Keys in Other DL-based Schemes}

There are many other discrete logarithm-based protocols which may be susceptible to our attack. In this section we present several such examples which we found in the literature. They include threshold cryptosystems based on ElGamal encryption [15], anonymous channels used in electronic voting schemes $[30,36]$ and undeniable signatures $[12,8,29]$.

\subsection{Shared Decryption of ElGamal Encryption}

ElGamal encryption of message $m$ for user $A$ consists of $\left\{c_{1}, c_{2}\right\}$, where $c_{1}=g^{k}$ $\bmod p$ with $k \in_{R} \mathbf{Z}_{q}$ and $c_{2}=m y_{A}^{k} \bmod p$ [18]. The receiver $A$ can decrypt the ciphertext $\left\{c_{1}, c_{2}\right\}$ by computing $m=c_{2} c_{1}^{-x_{A}} \bmod p$. In some group-oriented applications we may need to encrypt the message in such a way that only an authorized subset of receivers can decrypt the ciphertext. This can be done using ElGamal encryption and Shamir's secret sharing scheme [37].

As an example, we consider a prime field implementation of the threshold cryptosystem proposed by Desmedt and Frankel [15]. Let $\mathbf{G}$ be a group of $n$ members and $y_{G}=g^{x_{G}} \bmod p$ be a public key of the group. We want to encrypt a message $m$ so that any subset of $t$ or more members in $\mathbf{G}$ can read the message. For this, in the system setup phase a trusted authority picks a random polynomial $f$ of degree $t-1$ in $\mathbf{Z}_{q}$ such that $f(0)=x_{G}$, i.e., $f(z)=a_{t-1} z^{t-1}+\cdots+a_{1} z+x_{G}$ with $a_{j} \in_{R} \mathbf{Z}_{q}$, computes secret shares $x_{G i}=f(i) \bmod q$ for $i=1,2, \cdots, n$ and securely sends $x_{G i}$ to each member $i$ of $\mathbf{G}$. (See [24] for a more flexible scheme not requiring such pre-distribution of secret shares.) Now, suppose that a ciphertext $\left\{c_{1}, c_{2}\right\}$, where $c_{1}=g^{k} \bmod p$ and $c_{2}=m y_{G}^{k} \bmod p$, is received and that a subset $\mathbf{H}$ of $t$ members in $\mathbf{G}$ agreed to decrypt the ciphertext. Then each member $j \in \mathbf{H}$ computes $w_{j}=c_{1}^{-b_{j} x_{G j}} \bmod p$, where $b_{j}=\prod_{i \in \mathbf{H}, i \neq j} \frac{-i}{j-i} \bmod q$, and sends $w_{j}$ to a combiner (e.g., one designated member). The combiner then computes $w=\prod_{j \in \mathbf{H}} w_{j} \bmod p$, which should be $c_{1}^{-x_{G}} \bmod p$ if all members involved worked correctly. Therefore, the message $m$ can be recovered by $m=c_{2} w$ $\bmod p$.

It is easy to see that our attack can be successful for the above scheme, if each shareholder does not check that $c_{1}^{q}=1 \bmod p$. In this case, our attack can 
extract much more secret key bits at a time. Let $\gamma=\prod \beta_{i}$ (a product of smooth order elements). The attacker sends a ciphertext $\left\{c_{1}, c_{2}\right\}$ such that $c_{1}=\gamma g^{k}$ $\bmod p$ and $c_{2}=m y_{G}^{k} \bmod p$. Since $w_{j}^{q}=\left(\gamma^{q}\right)^{-b_{j} x_{G}} \bmod q \bmod p$, once obtaining $w_{j}$, he can easily compute the logarithm $\left(-b_{j} x_{G j} \bmod q\right) \bmod \operatorname{ord}(\gamma)$ using a Pohlig-Hellman decomposition. The remaining part of $-b_{j} x_{G j} \bmod q$ can be found from the value $y_{j}=g^{x_{G j}}=w_{j}^{\left(-b_{j} k\right)^{-1}} \bmod p$. This reveals the secret share of a shareholder $j$. If $w_{j}$ 's are transmitted through a secure channel, the attacker need to collude with the combiner.

Note the efficiency of the above attack. Unlike in key exchange protocols, where the attacker can only obtain a function of the shared secret (e.g., a hash value), in the above scheme the attacker has direct access to the shared secret itself (i.e., a value exponentiated with the secret key). This allows the attacker to get a Pohlig-Hellman decomposition for the secret key. Since now Pollard's $\rho$ method can be used to solve the decomposed problems, it would be quite feasible to use a $\beta$ of order about 80 bits. Thus, for a random prime $p$ such that $q \mid p-1$ and $|q|=160$, the attack could reveal the whole secret key in most cases.

Anonymous channels proposed by Park et al.[30] uses a special case of the threshold cryptosystem described above, i.e., the case of $t=n$. The anonymous channel is primarily used to protect the secrecy of votes in electronic voting schemes. Later Pfitzmann [32] developed successful attacks on these channels. To defeat such attacks, Sako and Kilian [36] used a prime order subgroup in their election scheme, instead of the full multiplicative group $\mathbf{Z}_{\mathbf{p}}^{*}$ originally used in [30]. However, in this case our attack can be applied again. To see this, we briefly describe the modified version in [36].

Each MIX $M_{i}(1 \leq i \leq n)$ has a secret key $x_{i} \in_{R} \mathbf{Z}_{q}$ and publishes its public key $y_{i}=g^{x_{i}} \bmod p$. Let $w_{j}=\prod_{i=j+1}^{n} y_{i} \bmod p$ for $j<n$ and $w_{n}=1$. For each ciphertext $C_{0}=\left\{c_{0,1}, c_{0,2}\right\}=\left\{g^{k}, m w_{0}^{k}\right\}$, each MIX $M_{i}$ for $i=1,2, \cdots, n-1$ transforms the $C_{i-1}$ posted by $M_{i-1}$ into $C_{i}=\left\{c_{i-1,1} g^{r_{1}}, c_{i-1,2} w_{i}^{r_{1}} c_{i-1,1}^{-x_{i}}\right\}$ with random $r_{i} \in \mathbf{Z}_{q}$ and posts the $C_{i}$ on the public board in alphabetical order (all computations are done in $\bmod p$ ). In [36] this is done in two phases: in the first phase $M_{i}$ posts $z_{i}=c_{i-1,1}^{x_{i}} \bmod p$ and in the second phase it posts $\left\{c_{i-1,1} g^{r_{i}}, c_{i-1,2} w_{i}^{r_{i}} / z_{i}\right\}$. In each phase $M_{i}$ also proves the correctness of the computation. Now the final MIX $M_{n}$ can recover $m$ by computing $c_{n-1,2} c_{n-1,1}^{-x_{n}}$ $\bmod p$.

As is clear, this protocol is vulnerable to our attack. A voter $V$ can submit a faked vote $C_{0}=\left\{\gamma c_{0,1} \bmod p, c_{0,2}\right\}$ and then find $x_{i} \bmod \operatorname{ord}(\gamma)$ from $z_{i}$ as before. Therefore, it is essential that each MIX $M_{i}$ should verify that $c_{i-1,1}^{q}=1$ $\bmod p$ before beginning its processing.

\subsection{Undeniable Signatures}

Our attack can also be applied to some digital signature applications. The most obvious case is to produce an undeniable signature on message $m \in \mathbf{Z}_{q}$ as $m^{x}$ $\bmod p$ without checking that $m^{q}=1 \bmod p$, where $x$ is the signer's secret key. We could find several other examples in the literature. 
As a first example, we consider the validator issuing protocol by Chaum and Pedersen (see Sec.4 in [13]). The purpose of this protocol is that a center $Z$ issues a validator to a 'wallet with observer' (consisting of a computer $C$ and a tamper-proof module $T$ embedded inside $C$ ). The validator is an unlinkable certificate for the public key $y_{T}=g^{x_{T}}$ of $T$. In some steps of the protocol the computer $C$ blinds $y_{T}$ as $m=y_{T}^{k} \bmod p$ with random $k$ and sends it to the center $Z$, who then returns $z_{0}=m^{x_{z}} \bmod p$. Obviously, if $C$ sends $m=\gamma y_{T}^{k}$ $\bmod p$ with $\gamma=\prod \beta_{i}$, it can find $x_{Z} \bmod \operatorname{ord}(\gamma)$ from $z_{0}^{q}=\left(\gamma^{q}\right)^{x_{Z}} \bmod p$ using the Pohlig-Hellman method. Note that $C$ can still obtain the desired signature by computing $z_{0} \gamma^{-x_{z}} \bmod p$ after finding $x_{Z} \bmod \operatorname{ord}(\gamma)$. The same attack can be applied to its privacy enhanced version [14] if the signer does not check that $m^{q}=1 \bmod p$. The authors may omit this checking step in the thought that $C$ can only obtain an undeniable signature for a random message, but this omission enables a fatal attack as shown above.

In Brands's electronic cash scheme using a wallet with observers [6] (see [7] for more details), each user computes $I=g_{1}^{u} \bmod p$ with $u \in \in_{R} \mathbf{Z}_{q}$ and sends it to the bank, which generates a signature $z=\left(I g_{2}\right)^{x} \bmod p\left(g_{1}, g_{2}\right.$ generators of a subgroup of order $q$ ). In this case the user must prove to the bank that he knows $u$ since $I$ corresponds to the account number of the user (see also [11]). Thus our attack is not applicable here. However, as noted in Sec.2.1, it is essential to check $I^{q}=1 \bmod p$ at the begining of the proof if a Schnorr-type identification scheme is used for this purpose (this is the case in [7]). Otherwise, the user can pass the proof with $I=\beta g_{1}^{u} \bmod p$ in success probability $1 / \operatorname{ord}(\beta)$. The successful pass will be fatal in this system: Not only the user can mount our attack to find partial information on the secret $x$, but also he can spend the same coin multiple times without being identified.

Another possibility for the attack exists in the confirmation protocol of undeniable signatures $[12,8]$ and designated confirmer signatures [29]. For example, consider the convertible undeniable signature scheme by Boyar et al.[8]. ${ }^{4}$ In this scheme the signer $S$ possesses two secret/public key pairs, $\left\{x \in_{R} \mathbf{Z}_{q}, y=g^{x}\right.$ $\bmod p\}$ and $\left\{z \in_{R} \mathbf{Z}_{q}, u=g^{z} \bmod p\right\}$. The signature on message $m$ is a triple $\{t, r, s\}$, where $t=g^{k_{1}} \bmod p, r=g^{k_{2}} \bmod p$ and $s=k_{2}^{-1}\left(h(m) t z k_{1}-x r\right) \bmod$ $q\left(k_{1}, k_{2} \in_{R} \mathbf{Z}_{q}\right)$. Thus the signature $\{t, r, s\}$ is valid iff $\left(t^{h(m) t}\right)^{z}=y^{r} r^{s} \bmod p$. The confirmation protocol between $S$ and $V$ is as follows:

1. $S$ and $V$ computes $w=t^{h(m) t} \bmod p$ and $v=y^{r} r^{s} \bmod p$ from the signature $\{t, r, s\}$.

2. $V$ computes a challenge $c h=w^{a} g^{b} \bmod p$ with $a, b \in_{R} \mathbf{Z}_{q}$ and sends $c h$ to $S$.

3. $S$ computes $h_{1}=c h \cdot g^{c} \bmod p$ with $c \in_{R} \mathbf{Z}_{q}$ and $h_{2}=h_{1}^{z} \bmod p$, and sends $\left\{h_{1}, h_{2}\right\}$ to $V$.

4. $V$ reveals $a$ and $b$ to $S$.

4 This scheme is taken as an example only to illustrate our attack, not to show that it is insecure. Michels et al. [28] have already shown that this scheme could be broken when used as a totally convertible signature scheme. We would like to thank one of anonymous referees for pointing out this attack. 
5. $S$ checks that $c h=w^{a} g^{b} \bmod p$. if it holds true, then $S$ reveals $c$ to $V$. Otherwise, $S$ stops the protocol.

6. $V$ checks that $h_{1}=w^{a} g^{b+c} \bmod p$ and $h_{2}=v^{a} u^{b+c} \bmod p$.

This protocol is complete, sound and proven zero-knowledge. However, suppose that the verifier $V$ sends, as a challenge in step 2, any value of order $q$ multiplied by small order elements, say $c h=\gamma g^{b} \bmod p$. Then the received value $h_{2}$ in step 3 satisfies $h_{2}^{q}=\left(\gamma^{q}\right)^{z} \bmod p$, from which the verifier can find $z$ $\bmod \operatorname{ord}(\gamma)$. This shows that the confirmation protocol cannot be zero-knowledge against a dishonest verifier, unless the prover checks that $c h^{q}=1 \bmod p$ in step 3. In a variant by Pedersen [31], $S$ computes $h_{1}, h_{2}$ as $h_{1}=(c h)^{c} \bmod p$ with $c \in_{R} \mathbf{Z}_{q}$ and $h_{2}=h_{1}^{z} \bmod p$. This variant is also vulnerable to our attack, since one can still obtain the equation $h_{2}^{q}=\left(h_{1}^{q}\right)^{z} \bmod p$ by sending $c h=\gamma g^{b} \bmod p$ (here note that $\left.\operatorname{ord}\left(h_{1}^{q}\right)=\operatorname{ord}(\gamma)\right)$.

The above attack suggests that a prime $p$ should be chosen as $p=2 q+1$ ( $q$ prime) if an undeniable signature is computed as $m^{x} \bmod p$ and if the above protocol is to be used for confirmation, as in Chaum's undeniable signature [12]. ${ }^{5}$ On the other hand, Jakobsson and Yung [19] proposed an oblivious decision proof protocol for proving validity/invalidity of undeniable signatures, where the prover does not have to know whether the signature in question is valid or not. Thus the protocol does not necessarily require the message $m$ to be in $\mathbf{Z}_{q}$. They choose the system parameters for use in Chaum's scheme as: $p=q l+1$ ( $p, q$ prime, $l$ integer), $g$ an element of order $q$ and $\left\{x \in_{R} \mathbf{Z}_{q}, y=g^{x} \bmod p\right\}$ as the secret/public key pair of the signer. However, careful examination shows that their oblivious protocol with such parameters is also vulnerable to our attack. This protocol cannot be repaired by simply adding checking steps as noted above. To avoid our attack, we have to choose $p$ as $p=2 q+1$ ( $q$ prime). Or we may use the full multiplicative group $\mathbf{Z}_{p}^{\star}$ as the underlying group.

We note that the signer or the confirmer(s) must verify the validity of a signature before executing the confirmation protocol if distinct protocols are used for confirmation and disavowal. ${ }^{6}$ Otherwise, the verifier may change the first component $t$ of the signature as $t^{\prime}=\gamma t \bmod p$ and requests $S$ to confirm its validity by sending $\left\{t^{\prime}, r, s\right\}$. Then $V$ will be able to obtain $\gamma^{z} \bmod p$ at the end of the protocol, since the check in step 5 will succeed if $V$ sends $c h=\left(w^{\prime}\right)^{a} g^{b}$ $\bmod p$ with $w^{\prime}=\left(t^{\prime}\right)^{t^{\prime} h(m)} \bmod p$ in step 2. If the secret $z$ is distributed into a set of designated confirmers [31,29], an authorized subset of confirmers should run a shared verification protocol to validate the signature. Then there may exist

\footnotetext{
$\overline{5}$ Note that it is infeasible to generate $m$ as an element of order $q$ for any meaningful message or its hash value if $q$ is chosen small compared to $p$. Thus there is no way to detect our attack unless $p$ is chosen as $p=2 q+1$. Alternatively, we may choose $g$ as a generator of $\mathbf{Z}_{p}^{\star}$. Then our attack will be useless; the public key itself already reveals the smooth part of the secret key.

6 There is another weakness when the validity of a signature is not verified before confirmation, as described in Appendix A in [19]: If a verifier who does not have a valid signature for $m$ is allowed to participate in the confirmation protocol, then the verifier can easily obtain a valid signature for $m$.
} 
another possibility for the combiner to mount a similar attack to find secret shares of confirmers as in the shared decryption of ElGamal ciphertexts (see Sec.3.1), unless each confirmer checks that $w^{q}=1 \bmod p$.

\section{Generating and Registering Public Parameters}

The presented attack shows that it is essential in discrete log-based schemes using a prime order subgroup to check the order of received numbers before applying the secret key. However, such explicit checking requires additional exponentiation. As a better countermeasure, we recommend to use a secure prime, i.e., a prime $p$ such that $(p-1) / 2 q$ is also prime or each prime factor of $(p-1) / 2 q$ is larger than $q$. (see also [25] for a method of generating primes which can substantially reduce the modular reduction time and storage usage). Such a prime can be generated much faster than, and seems as strong against any known attack as, a safe prime (i.e., a prime of the form $p=2 q+1$ ).

To generate a prime $p$ such that $p=2 q p_{1}+1$, we first choose a random prime $p_{1}$ of length $|p|-|q|-1$ and then find a desired prime $p$ by testing $p=2 q p_{1}+1$ for primality with random primes $q$ 's of size $|q|$. Thus we need to generate a number of $q$ 's to find a $p$ (e.g., about 710 for a 1024-bit prime $p$, considering the density of primes, $1 / \ln x$ ). However, this does not require much time, since the size of $q$ is quite small compared to that of $p$ (usually $|q|=160$ and $|p|=768$ or 1024).

It is much cheaper to generate a prime $p$ such that $p=2 q p_{1} p_{2} \cdots p_{n}+1$, where $p_{i}$ 's are primes almost equal to $q$. We first determine the number $n$ from the inequality $l=\left|p_{i}\right| \approx(|p|-|q|-1) / n \geq|q|$. Then we generate a pool of primes for $p_{i}$ 's. Suppose that the pool contains $m$ primes of size $l$. Then we have $\left(\begin{array}{l}m \\ n\end{array}\right)$ candidates for $p$. Considering the density of primes, we can make this number large enough to guarantee that there are enough possibilities for a prime $p$ to be found with this prime pool. For example, for a 1024-bit prime $p$ and a 160-bit prime $q$ we may choose $l \approx 173, n=5$ and $m=15$. This choice of parameters gives about 3000 candidates for $p$. Thus testing this many candidates will produce a prime $p$ with very high probability.

There are other advantages in our proposed prime generating methods. They give a complete factorization of $p-1$, which may be useful if we need to find a primitive element $\bmod p .^{7}$ Furthermore, since $p-1$ contains many prime factors of similar size, we may use different subgroups of prime order for different applications (e.g., one for ordinary signatures, one for undeniable signatures and one for key exchange, etc.). This will prevent any potential weakness from the misuse of key parameters. Of course, using the same prime $p$ in different primitives may not be desirable in view of security against most discrete logarithm algorithms, such as the index calculus method and the number field sieve. However, this gives us efficiency in storage and communication.

${ }^{7}$ Note that there is no known algorithm to find a primitive element $\bmod p$ without knowing the factorization of $p-1$. The simplest is to check the order of elements successively (say, $2,3,5, \cdots$ ) using the prime factors of $p-1$. 
The attacks presented in this paper and in Menezes et al.[27] show that the CA must check for knowledge of a secret key corresponding to the public key before issuing a certificate. In particular, the CA and each user must first check the order of the received base $g$ and public key $y$. If such an interactive proof is hard to carry out in some environments, we propose to use the following certification procedure, which seems to preclude any known weakness even without such a proof. The basic idea is to allow the CA to also contribute to the randomness of a user's secret key. On receiving a user's part of a public key $y^{\prime}=g^{x^{\prime}} \bmod p$, the CA computes the actual public key $y$ as $y=\left(y^{\prime}\right)^{a} g^{b} \bmod p$, where $a=\frac{p-1}{q} c$ and $b, c \in_{R} \mathbf{Z}_{q}$. The CA now generates a certificate for $y$ and sends $\{a \bmod q, b\}$, along with the certificate, to the user. On receiving $\{a, b\}$, the user checks that $a \neq 0 \bmod q$, computes the actual secret key $x$ as $x=x^{\prime} a+b \bmod q$ and finally checks that the certified public key $y$ is equal to his own computation $g^{x} \bmod p$.

The exponent $a$ makes vanish any component of $y^{\prime}$ which does not belong to the subgroup of order $q$. This prevents users from registering an improper form of public keys. The multiplicative factor $g^{b} \bmod p$ makes it impossible for a user to register a public key as a power of other user's public key. This prevents the attack presented in [27]. Furthermore, users' secret keys can be made more pseudorandom by the CA's contribution if the CA is equipped with a true random generator or uses a cryptographically strong pseudorandom generator (in this case we assume that the CA sends $\{a \bmod q, b\}$ through a secure channel). This may be advantageous since most users may not be so careful in choosing their secrets.

\section{Conclusion}

We have demonstrated that many discrete logarithm protocols may be insecure against a key recovery attack unless suitable checking steps are added. The presented attack may reveal part of, in many cases the whole, secret key of a victim in a reasonable time for many DL-based schemes published in the literature. The attack exploits small order subgroups in $\mathbf{Z}_{p}^{*}$ to compute part of the secret key in a protocol working in a subgroup of prime order $q$. This is possible since in most schemes a prime $p$ is chosen at random such that $q \mid p-1$. Therefore, our attack can be easily prevented if relevant protocol variables are properly checked, that is, if each party checks that received numbers belong to the underlying subgroup of prime order. However, such explicit checkings substantially decrease efficiency. Thus a better alternative would be to minimize possible leakage of secret key bits by using a secure prime, a prime $p$ such that all prime factors of $(p-1) / 2 q$ are larger than $q$. Such a prime only leaks one bit of the secret by our attack.

\section{References}

1. R.Anderson and R.Needham, Robustness principles for public key protocols, In Advances in Cryptology - CRYPTO'95, LNCS 963, Springer-Verlag, 1995, pp.236247. 
2. R.Anderson and S.Vaudenay, Minding your $p$ 's and $q$ 's, In Advances in Cryptology - ASIACRYPT'96, LNCS 1163, Springer-Verlag, 1996, pp.15-25.

3. A.Aziz, T.Markson and H.Prafullchandra, Simple key-management for Internet protocols (SKIP), draft-ietf-ipsec-skip-07.txt, Aug. 1996. (see also the SKIP home page $h t t p: / / s k i p . i n c o g . c o m /$ for more information.)

4. D.Bleichenbacher, Generating ElGamal signatures without knowing the secret, In Advances in Cryptology - EUROCRYPT'96, LNCS 1070, Springer-Verlag, 1996, pp.10-18.

5. C.Boyd and W.Mao, Design and analysis of key exchange protocols via secure channel identification, In Advances in Cryptology - ASIACRYPT'94, LNCS 917, Springer-Verlag, 1995, pp.171-181.

6. S.Brands, Untraceable off-line cash in wallet with observers, In Advances in Cryptology - CRYPTO'93, LNCS 773, Springer-Verlag, 1994, pp.302-318.

7. S.Brands, An efficient off-line electronic cash system based on the representation problem, Technical Report CS-R9323, CWI, Amsterdam, 1993.

8. J.Boyar, D.Chaum, I.Damgard and T.Pedersen, Convertible undeniable signatures, In Advances in Cryptology - CRYPTO'90, LNCS 537, Springer-Verlag, 1991, pp.189-205.

9. M.Burmester, A remark on the efficiency of identification schemes, In Advances in Cryptology - EUROCRYPT'90, LNCS 473, Springer-Verlag, 1991, pp.493-495.

10. M.Burmester, On the risk of opening distributed keys, In Advances in Cryptology - CRYPTO'94, LNCS 839, Springer-Verlag, 1994, pp.308-317.

11. A.Chan, Y.Frankel and Y.Tsiounis, Mis-representation of identities in E-cash schemes and how to prevent it, In Advances in Cryptology - ASIACRYPT'96, LNCS 1163, Springer-Verlag, 1996, pp.276-285.

12. D.Chaum, Zero-knowledge undeniable signatures, In Advances in Cryptology - EUROCRYPT'90, LNCS 473, Springer-Verlag, 1991, pp.458-464.

13. D.Chaum and T.Pedersen, Wallet databases with observers, In Advances in Cryptology - CRYPTO'92, LNCS 740, Springer-Verlag, 1993, pp.89-105.

14. R.Cramer and T.Pedersen, Improved privacy in wallets with observers, In Advances in Cryptology - EUROCRYPT'93, LNCS 765, Springer-Verlag, 1994, pp.329-343.

15. Y.Desmedt and Y.Frankel, Threshold cryptosystems, In Advances in Cryptology CRYPTO'89, LNCS 435, Springer-Verlag, 1990, pp.307-315.

16. W.Diffie and M.E.Hellman, New directions in cryptography, IEEE Trans. Info. Theory, 22(6), 1976, pp.644-654.

17. W.Diffie, P.van Oorschot and M.Wiener, Authentication and authenticated key exchange, Designs, Codes and Cryptography, 2, 1992, pp.107-125.

18. T.ElGamal, A public key cryptosystem and a signature scheme based on discrete logarithms, IEEE Trans. Inform. Theory, IT-31, 1985, pp.469-472.

19. M.Jakobsson and M.Yung, Proving without knowing: on oblivious, agnostic and blindfolded provers, In Advances in Cryptology - CRYPTO'96, LNCS 1109, Springer-Verlag, 1996, pp.186-200.

20. M.Just and S.Vaudenay, Authenticated multi-party key agreement, In Advances in Cryptology - ASIACRYPT'96, LNCS 1163, Springer-Verlag, 1996, pp.36-49.

21. H.Krawczyk, SKEME: A versatile secure key exchange mechanisms for Internet, In Proc. of 1996 Symp. on Network and Distributed Systems Security.

22. A.K.Lenstra, P.Winkler and Y.Yacobi, A key escrow system with warrant bounds, In Advances in Cryptology - CRYPTO'95, LNCS 963, Springer-Verlag, 1995, pp.197-207.

23. C.H.Lim and P.J.Lee, Several practical protocols for authentication and key exchange, Information Processing Letters, 53, 1995, pp.91-96. 
24. C.H.Lim and P.J.Lee, Directed signatures and application to threshold cryptosystems, In Pre-Proc. of 1996 Cambridge Workshop on Security Protocols, The Isaac Newton Institute, Cambridge, April 1996.

25. C.H.Lim and P.J.Lee, Generating efficient primes for discrete log cryptosystems, submitted for publication (also presented at ASIACRYPT'96 Rump Session).

26. T.Matsumoto, Y.Takashima and H.Imai, On seeking smart public-key distribution systems, The Transactions of the IEICE of Japan, E69, 1986, pp.99-106.

27. A.J.Menezes, M.Qu and S.A.Vanstone, Some new key agreement protocols providing implicit authentication, In Proc. SAC'95, Carleton Univ., Ottawa, Ontario, May 1995, pp.22-32.

28. M.Michels, H.Petersen and P.Horster, Breaking and repairing a convertible undeniable signature scheme, Proc. of 3rd ACM Conference on Computer and Communications Security, Mar. 1996.

29. T.Okamoto, Designated confirmer signatures and public-key encryption are equivalent, In Advances in Cryptology - CRYPTO'94, LNCS 839, Springer-Verlag, 1995, pp.61-74.

30. C.S.Park, K.Itoh and K.Kurosawa, Efficient anonymous channel and all/nothing election scheme, In Advances in Cryptology - EUROCRYPT'93, LNCS 765, Springer-Verlag, 1994, pp.248-259.

31. T.Pedersen, Distributed provers with applications to undeniable signatures, In Advances in Cryptology - EUROCRYPT'91, LNCS 547, Springer-Verlag, 1991, pp.221-242.

32. B.Pfitzmann, Breaking an efficient anonymous channel, In Advances in Cryptology - EUROCRYPT'94, LNCS 950, Springer-Verlag, 1995, pp.332-340.

33. S.C.Pohlig and M.E.Hellman, An improved algorithm for computing logarithms over $G F(p)$ and its cryptographic significance, IEEE Trans. Inform. Theory, IT-24 (1), 1978, pp.106-110.

34. D.Pointcheval and J.Stern, Security proofs for signature schemes, In Advances in Cryptology - EUROCRYPT'96, LNCS 1070, Springer-Verlag, 1996, pp.387-398.

35. J.M.Pollard, Monte Carlo methods for index computation $(\bmod p)$, Math. Comp., 32(143), 1978, pp.918-924.

36. K.Sako and J.Kilian, Receipt-free mix-type voting scheme, In Advances in Cryptology - EUROCRYPT'95, LNCS 921, Springer-Verlag, 1995, pp.pp.393-403.

37. A.Shamir, How to share a secret, Commun. ACM, 22, 1979, pp.612-613.

38. C.P.Schnorr, Efficient identification and signatures for smart cards, In Advances in Cryptology - CRYPTO'89, LNCS 435, Springer-Verlag, 1990, pp.235-251.

39. J.Stern, The validation of cryptographic algorithms, In Advances in Cryptology ASIACRYPT'96, LNCS 1163, Springer-Verlag, 1996, pp.301-310.

40. P.C.van Oorschot and M.J.Wiener, Parallel collision search with applications to hash functions and discrete logarithms, In Proc. 2nd ACM Conference on Computer and Communications Security, Fairfax, Virginia, Nov. 1994, pp.210-218.

41. P.C.van Oorschot and M.J.Wiener, On Diffie-Hellman key agreement with short exponents, In Advances in Cryptology - EUROCRYPT'96, LNCS 1070, SpringerVerlag, 1996, pp.332-343.

42. S.Vaudenay, Hidden collisions on DSS, In Advances in Cryptology - CRYPTO'96, LNCS 1109, Springer-Verlag, 1996, pp.83-88.

43. Y.Yacobi, A key distribution paradox, In Advances in Cryptology - CRYPTO'90, LNCS 537, Springer-Verlag, 1991, pp.268-273.

44. ISO/IEC JTC1/SC27, Information technology - Security techniques - Key management - Part 3: Mechanisms using asymmetric techniques. 